\title{
Value of High-Frequency Relic Gravitational Wave (HFRGW) Detection to Astrophysics and Fabrication and Utilization of the Li-Baker HFRGW Detector
}

\author{
Andrew Walcott Beckwith1, Robert M. L. Baker Jr² \\ ${ }^{1}$ Physics Department, College of Physics, Chongqing University Huxi Campus, Chongqing University, Chongqing, China \\ ${ }^{2}$ Transportation Sciences Corporation, Palm Desert, CA, USA \\ Email: rwill9955b@gmail.com, abeckwith@uh.edu,DrRobertBaker@GravWave.com
}

How to cite this paper: Beckwith, A.W. and Baker Jr, R.M.L. (2020) Value of High-Frequency Relic Gravitational Wave (HFRGW) Detection to Astrophysics and Fabrication and Utilization of the Li-Baker HFRGW Detector. Journal of High Energy Physics, Gravitation and Cosmology, 6, 103-122. https://doi.org/10.4236/jhepgc.2020.61010

Received: October 23, 2019

Accepted: January 17, 2020

Published: January 20, 2020

Copyright $\odot 2020$ by author(s) and Scientific Research Publishing Inc. This work is licensed under the Creative Commons Attribution International License (CC BY 4.0).

http://creativecommons.org/licenses/by/4.0/ (c) (i) Open Access

\begin{abstract}
Up to the present time gravitational-wave detectors, such as LIGO and Virgo, have been sensitive to frequencies on the order of a few thousand to a small fraction of an Hz. They have been most effective in the study of black-hole mergers. We suggest that high-frequency relic gravitational wave (HFRGW) detectors be developed, especially the Li-Baker HFRGW detector, in the gigahertz and higher frequency range. We believe collecting cosmological, primordial observational data especially generated during the first few seconds after the beginning of our Universe is extremely important. One motivation for this paper is, therefore, that we are confident that observation of relic gravitational waves will provide vital information about the birth of our Universe and its early dynamical evolution. Other astrophysical applications of HFRGW detectors involve the entropy growth of the early Universe, an ability to study alternatives to inflation and to provide clues about the symmetries underlying new physics at the highest energies. A working hypothesis or theory, based upon the rollout of our Universe from infinitesimal Planck Length and Planck Time is presented. This theory involves the rapid motion of time and matter during that early time having frequencies on the order of trillions of cycles per second or more. Several alternative HFRGW detectors are described and the proposed Li-Baker HFRGW detector, which is theoretically sensitive to GW amplitudes, A, as small as $10^{-32}$, is discussed in detail. Such sensitivity may provide a means for verifying or falsifying the rollout of our Universe working hypothesis. Essentially a combination of theory and experimentation is presented. It is recommended that plans and detailed specifications for the Li-Baker HFRGW detector be prepared in order to expedite
\end{abstract}


its fabrication.

\section{Keywords}

High-Frequency Gravitational Waves, Dark Energy, Dark Matter, Variation of Speed of Time, Beyond the Standard Model of Cosmology, Early Universe

\section{Introduction}

A detection of the special pattern produced by gravitational waves would be not only an unprecedented discovery, "... but also a direct probe of physics at the earliest observable instants of our Universe"-from The Origin of the Universe as Revealed Through the Polarization of the Cosmic Microwave Background [1]. This is the traditional treatment but Grishchuk (2006) [2] found the energy density of relic gravitational waves is given by

$$
\Omega_{g w}(v)=(\pi / 3) h^{2}(v)\left(v / v_{H}\right)^{2}
$$

where $\Omega_{g w}(v)$ is the energy density, $h(v)$ is the amplitude of the gravitational wave having frequency $v$ and $\left(v / v_{H}\right)$ is the ratio of that frequency to a specific gravitational-wave frequency $v_{H}$.

Shown in Figure 1 and Figure 2, is the detection sensitivity required, $h_{r m s}$ as a function of HFRGW frequency. The parameter $n$ in Figure 1 and Figure 2 is defined as the slope of the primordial GW spectrum. In addition, we have that Figure 3 defines a change in times slope which is important and can be investigated by high frequency GW data sets, whereas Figure 4 is that of the Birmingham

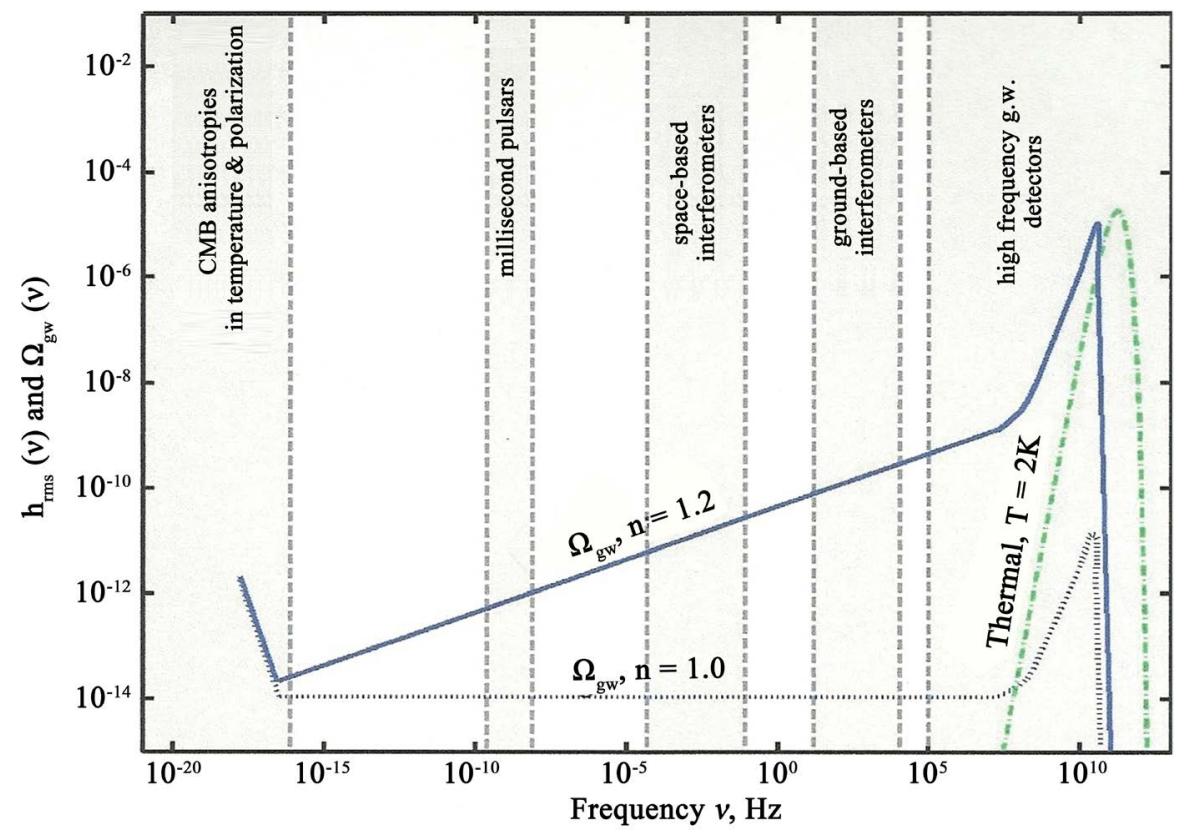

Figure 1. Predicted relic gravitational wave energy density as a function of frequency (Grishchuk, 2008) [3]. 


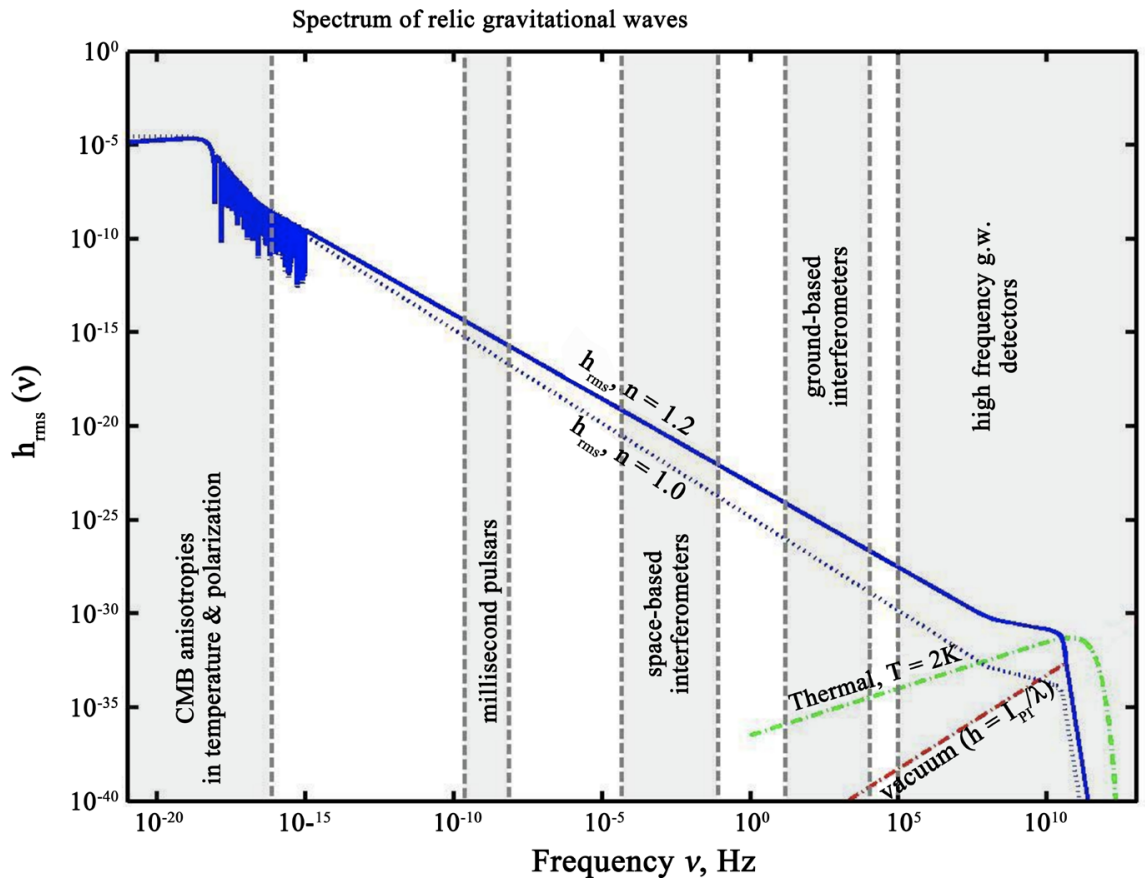

Figure 2. Spectrum of relic gravitational wave amplitudes as a function frequency (Grishchuk, 2008).

GW detector which is yet another way to obtain HFGW. In addition, the parameter $n$, as stated in relevance to Figure 1 and Figure 2 is accessible for measurement directly in Figure 5 and Figure 6, i.e. see (Grishchuk, 2008) [3] with $n$ having a value whose exact value is currently unknown but which is thought to be between 1.0 and 1.2. Measuring the HFRGW spectrum would allow its value to be evaluated much more accurately. Grishchuk (2007) [4] showed that observation of relic gravitational waves to measure this parameter will enable significant direct inferences to be drawn about the value of the Hubble parameter (essentially the separation speed of cosmic objects, possibly due to the speed of time) of the early universe and the cosmological scale factor, both of which provide vital information about the birth of the Universe and its early dynamical evolution.

\section{Value of HFRGW Detection to Astrophysics}

Grishchuk (2007) [4] showed that the energy density of the relic HFGW is greatest at around $10 \mathrm{GHz}$ and the amplitude sensitivity required for detection at that frequency is about $\mathrm{A} \sim 10^{-30}$. The problem is that HFGWs at such a high frequency cannot be detected by the existing low-frequency gravitational wave detectors such as LIGO, GEO600 and Virgo or the planned Advanced LIGO, Big Bang Observer and LISA detectors, which are limited to a maximum frequency of a few kHz. For example, the advertised "high-frequency" range for maximum sensitivity of the Laser Interferometer Gravitational Observatory (LIGO) is 40 $\mathrm{Hz}$ to $2000 \mathrm{~Hz}$ (Shawhan, 2004 [5]; Shoemaker, 2008 [6]). The problem with higher frequencies is that the interference pattern between the LIGO legs, which 
is caused by the passage of a gravitational wave, must be observed. However, “... at higher frequencies, the quantum nature of the laser beam (made of discrete photons, albeit a large number of them) limits the precision of the measurement. Increased laser power would reduce the problem of quantum noise, but ultimately the LIGO (and other) interferometers (such as the Advanced LIGO and the proposed Laser Interferometer Space Antenna or LISA) are not suited to measuring gravitational waves that stretch or shrink the detector's arms much more rapidly than the time a photon typically remains in the optical cavity, which is roughly a millisecond for these interferometers (thus about a one kilocycle frequency upper limit)" (Shawhan, 2004) [5].

Another motivation for this paper is the analysis of entropy by means of high-frequency gravitational-wave detection. With regards to entropy, as a generalized astrophysical application, it can be thought of as ".. due to 'ignored' degrees of freedom, classically, and is generalized in general relativity by appealing to extreme entropy for all the null surfaces of space time." This last quote is from Thanu Padamanadan's address on this topic in the 25th IAGRG meeting in the Saha Institute in Calcutta, India, as brought up in Beckwith (2009a [7], 2009b [8] and 2009c [9]). Also, we should note that the entropy creation due to the possible presence of Dark Matter (DM) is different from accelerated entropy growth created by "relic gravitons" which is discussed in Beckwith (2008) [10] and DM entropy is meant as an initial entropy background prior to the Cosmic Microwave Background or CMBR barrier, i.e., graviton entropy generation is due to relic conditions, and should be thought of in line with a refinement of Jack Ng's work on DM entropy [11]. Beckwith earlier on, used Ngs infinite quantum statistics, [12] [13] Furthermore, as mentioned in Beckwith (2008) [10] there is a short-hand way to reference comparing graviton production via relic conditions, and neutrino physics, of the sort which can be measured in ICE CUBE data sets. As mentioned by Dr. Steinhardt of Princeton, to Beckwith (2008) [10] for relic gravitons, there are 105 times more relic neutrinos one can expect to observe in data sets as opposed to an individual relic graviton. A further review of some of these topics is in Beckwith's (2007) arXIV paper [14].

\section{Value of HFRGW Detection to Cosmology}

The Li-Baker detector (Baker et al., 2008 [15] and Li et al. 2008 [16]), especially due to its HFRGW sensitivity, would be a way to help establish a linkage to early universe theories, and neutrino physics. The importance of such a linkage cannot be over stated. And we have a chance with application of the Li-Baker detector to study challenges to the theory of cosmological inflation such as the rollout of spacetime. Cosmic microwave background polarization offers an extraordinary opportunity to gain a first glimpse into the physics that shaped our Universe. Experimentalists have demonstrated that a coordinated attack on this problem over the coming decade will likely require the detection of primordial 
gravitational waves - thereby providing extensive information about new physics at ultra-high energy scales.

The theory of how to obtain such gravitational wave/graviton "signatures" in the CMBR itself has been worked out in minute detail. Fangyu Li of Chongqing University (Li and Nan, 2009) [17] has the following abstract quote we cite due to its cogency as to presenting how to measure HFRGW signatures:

The displaying condition of strength, phase and polarization states of the high-frequency relic gravitational waves (HFRGWs) in the electromagnetic (EM) detecting systems is studied. It is shown that the displaying condition depends not only on sensitivity of the EM detecting systems and the amplitudes of the HFRGWs, but also on the phase, the polarization states of the HFRGWs and their matching to the EM detecting systems. In order to display simultaneously the strength, phase and polarization states of the resonant "monochromatic component" of the HFRGWs, an important necessary condition would be utilization of two or multiple different EM detectors.

Given that $E$ and $B$ field modes are used already to mimic GW in the first place, what Li and Yang Nan (Li and Nan, 2009) [17] are offering is an analytical blueprint as to making precise detection for HFRGW by noting that "Since the frequencies $\left(10^{9}-10^{12} \mathrm{~Hz}\right)$ of HFRGW in the microwave band are much higher than that of the usual celestial GWs, and their dimensionless amplitudes may be only $10^{-34}-10^{-10} / \mathrm{rms} \mathrm{h} \mathrm{Hz}$, thus suitable detecting scheme to the HFRGW would be special EM resonance systems and not usual GW detectors such as LIGO, Virgo et al.".

In addition to the development of a technique to develop a new GW detection tool, our motivation is to develop a theory for the growth of our Universe that may be simpler and not require the presence of dark matter or dark energy. A simpler theory that may not require "that the nascent universe passed through a phase of exponential expansion soon after the Big Bang, driven by a positive vacuum energy density" (Lemley, 2002) [18]. Contemporary theories of our Universe, such as the Friedmann-Lemaitre-Robertson-Walker (FLRW) model of the cosmos (Brandenberger, 2011) [19], assume that time marches on at a uniform, constant pace.

\section{But What If That Is Not the Case?}

It is posited here that at the beginning of time, "Planck time" or "time zero" the speed of time was arbitrarily large according to our working hypothesis. One can imagine that the slowdown of time's "speed" as similar to having a malfunctioning wristwatch that is getting slower and slower each day. Each day it is getting worse and worse (slower and slower) until at last it has slowed to a near stop, the second hand is almost fixed at the "end of time" in our Universe. A working hypothesis [20] is defined as a hypothesis that is provisionally accepted as a basis for further research in the hope that a tenable theory will be produced, even if 
the hypothesis ultimately fails or is significantly modified (Isaac Newton's Principia Mathematica [21] [22], as significantly modified by Einstein [23], is an example). It is essentially an encouragement for further research and analyses.

This leads up now for an expansion of preliminary work done by Dr. Baker, which started in the works given in [24] and [25].

Essentially, we express our working hypothesis or theory is that our Universe is a rollout in space and time, spacetime, from the shortest length, Planck Length, (the smallest measure of length because shorter than it, quantum effects dominate) and the shortest meaningful measure of time, Planck Time (the time it would take a photon travelling at the speed of light to across a distance equal to the Planck Length). It is speculated that space dimensions grow very rapidly at first and that time also grows very rapidly at first. As time increases, the rate of its change could be erratic, that is although in general it slows (rate of time slows approaching zero at the end of time), its rate of change could decelerate, pause or perhaps accelerate for a while since there is no ap reason for constancy. Prior research includes course lectures by Baker in the 1970s through the early 1990s at UCLA, West Coast University and the United States Air Force Academy during which the "rollout theory" was developed and presented. The first written publication is to be found on pages 89-91 and 229 of (Baker, 2017) [26] and the invited poster presentation at the 2018 Annual Meeting of the American Association for the Advancement of Science or AAAS (Baker, 2018) [27]. José M. M. Senovilla, of the University of the Basque Country, Spain [28], theorized that the expansion of our Universe is an "illusion" and actually is the result of the higher speed of time during the period when the light left the stellar structures in the past,: "... we are fooled into thinking that the expansion of the Universe is accelerating because time itself is slowing down" (Mars, et al. 2008) [29]. So that according to Senovilla (2007) [28], the speed of time may be related to the "illusions" of dark matter and dark energy estimates. The reason that we have not been able to detect dark matter may just be that it does not exist.

The speed of time is essentially the independent, observable variable in our working hypothesis. A notional graph of the speed of time variation is shown in Figure 3. The relationship between the change in the speed of time and the change in the space dimensions is important since they rollout in concert-in fact, it is the fundamental equation of the working hypothesis! Its derivation comes directly from the previously presented and well-known definitions of Planck Length and Planck Time. At the beginning of our Universe, the (change in the space dimensions) divided by the (change in the speed of time) is equal to (zero to the Planck Length during Planck Time) divided by (zero to Planck Time during Planck Time), which equals the (speed of light). It is speculated that this relationship is correct and endures so that the fundamental equation is:

(change in the space dimensions)

$=($ speed of light $) \times($ change in the speed of time dimension $)$

In essence, it is speculated that quantum mechanics is the boundary or initial 
condition for our general-relativity behaving Universe of today. It is also speculated that the beginning of our Universe could be the osculating point with other universes (Baker and Noble, 2000) [24].

Similar to other cosmological theories, especially of the early universe, they should agree with observations, especially agree with new HFRGW observations yet to be taken. Since our working hypothesis suggests that the pace of time was faster in the past, galaxies would appear to rotate faster and stellar material would vary their rate of separation, the Hubble number (both essentially measurable observables of the speed of time). Therefore our working hypothesis must be shown to be compatible with both of these observations as it is with observations of a relationship between redshift and the inferred distance of faraway galaxies, the existence of a microwave background with a blackbody spectrum, etc. Most importantly, there would be no reason to introduce extraneous features to our Universe such as dark matter (DM) and/or dark energy, both of which have defied detection. Our working hypothesis must be shown by new HFRGW observations and resulting new theory also to be compatible with the assumption of homogeneity and isotropy of space and primarily relates to phenomena occurring less than a second after the beginning of time. Specifically, we are interested in time increasing from Planck Time (about $5.39 \times 10^{-44}$ seconds), the rollout speed of space dimensions (slowing from near the speed of light, about $3 \times 10^{8}$ meters per second), but increasing from Planck Length (about 1.6 $\times 10^{-35} \mathrm{~m}$ ) and the speed of time consequently, approaching 1.0 seconds per second. The situation with regard to the speed of time is described in the notional Figure 3, page 71 of Baker, 2019 [30] repeated here as Figure 3. To be

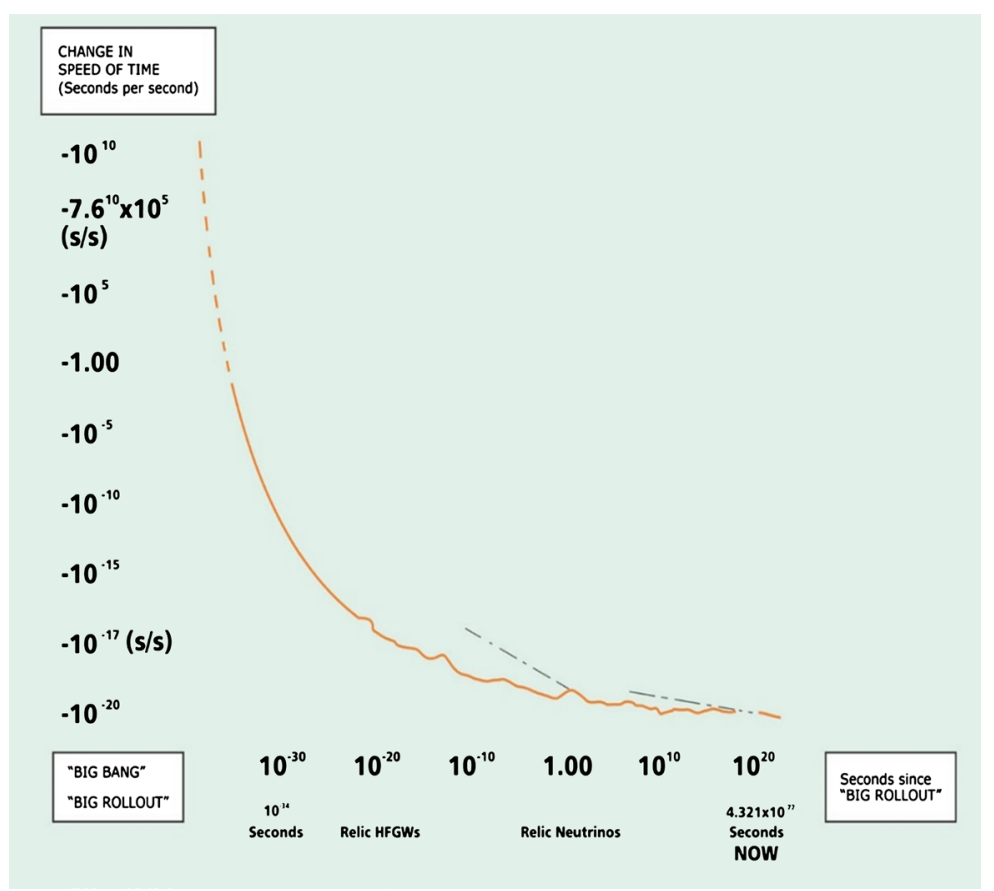

Figure 3. Notional graph of the change-of-speed-of-time variation with today's time dimension. Notice different slopes (tangents) and irregularities [30]. 
sure theoretical mathematical rigor must await such observations obtained less than a second or so after the beginning of our Universe, for example from observational data expected to be obtained from the Li-Baker HFRGW detector. That is, rigorous theory must await observations that show the actual variation of the speed of time to replace the notional graph of Figure 3.

According to the working hypothesis, in all frames of reference the speed of light is constant and the "laws of Physics" apply-a second is a second and a year is a year, (Baker, 2019, p. 71) [30] but as we look back in time through a telescope a second and a year, as well as distance measurements, appear shorter! (Baker and Baker, 2019 [31] for a video that includes the working hypothesis). In order to verify or falsify the working hypothesis, it would be useful to make observations at very short times, even a picosecond or less after "time zero". For that reason, Terahertz or even higher frequency HFRGW observations would be extremely valuable.

Viewing processes at past times today would see them moving with different rates depending upon the speed of time "back then." For example, if the Cosmic Microwave Background (CMB) occurred about 400,000 years (in length of today's years) after the beginning of our Universe, then we would see separation motion of the cosmos commensurate with the speed of time then, specifically, $(6.75 \pm 0.05) \times 10^{4} \mathrm{~m} / \mathrm{s}$ per Mpc (Alam, 2017) [32]. According to our working hypothesis, this separation speed (essentially the Hubble "constant") would be far smaller than separation speeds and, hence the speed of time far smaller than at a time closer to the beginning of our Universe. This speculation is to be tested by the measurement of HFRGWs. We also speculate that although the speed of time slows down tremendously after the beginning of our Universe, it could pause or even increase later on. Specifically, several billion years (today's years) after the beginning of our Universe (please see Figure 3) could have increased somewhat to $(7.4 \pm 1.5) \times 10^{4} \mathrm{~m} / \mathrm{s}$ per Mpc (Riess, 2019) [33]. Therefore, reflecting a slight increase in the speed of time. No "Dark Energy" needs to be assumed. Samuel Herrick back in the 1950s had an idea. He effectively incorporated the mass of an orbiting object, the mass of the object that is being orbited about and the Universal constant of gravity, $G$, into the time he often called "Tau Time" (Herrick, 1971) [34]. For this reason, whether the mass is associated with regular or Dark Matter is not of consequence. Of course, Herrick had no knowledge of "Dark Matter." As far as galactic motion is concerned, if one can incorporate the concept of "matter" or "mass" into a two-body orbital relationship, then multi-body or "n-body" motion, such as a galaxy would follow. The "Tau Time" for geocentric, heliocentric and planetocentric motion can be found in Table 1.1, page 24 of (Baker and Makemson, 1967) [35] along the row for "Time." In the working hypothesis on time, galactic motion can be predicted utilizing a speed of time local to a galaxy (like "Tau Time") without the need for Dark Matter (DM). That is, you determine the speed of time as the independent-variable observable as fitted to the measurements of the galactic motion using differential correction (Baker, 1967) [36]. 
As questioned on pages 68 and 69 of Baker (2019) [30]: How does the speed of time vary with time itself and is there a detailed structure to that change? Does the speed of time change depend upon location and "surroundings" in our Universe e.g., is it unique to the Earth (that is, change depending on location), change with the density of local matter, etc. and if so what is the relationship? What is the actual theory for the change of the speed of time, that is, what is its cause? Is there a starting point for time? Why is the direction of the time arrow in a single direction? Are there two opposite directions of time flow? Questions seeking answers that maybe be obtained from HFRGW-detector observations.

\section{Alternative HFRGW Detectors}

Let us consider alternative HFRGW detectors. One of the first suggested means for the detection of HFRGWs concerns electromagnetic detectors (Braginsky, et al. 1974) [37]. Then Pegoraro, et al. (1978) [38] suggested the use of tuned resonant chamber HFGW detectors. Rudenko and Sazhin in 1980 [39] proposed a Laser interferometer as a gravitational wave detector (somewhat similar to the current Japanese approach). In 1995 Tobar characterized multi-mode resonant-mass HFGW detectors [40] and three years later in 1998 Ottawa, et al. proposed a compact injection-locked Nd:YAG laser for HFGW detection. And in 1999 Tobar suggested, microwave parametric transducers for the next generation of resonant-mass gravitational wave HFRGW detectors [41].

In the past several years, HFRGW detectors have been fabricated at Birmingham University, England, INFN Genoa, Italy and in Japan. These types of detectors may be promising for the detection of the HFRGWs in the GHz band ( $\mathrm{MHz}$ band for the Japanese) in the future, but currently, their sensitivities are orders of magnitude less than what is required for the detection of HFRGWs from the" big bang" or "big rollout".

The Birmingham HFRGW detector shown in Figure 4 measures changes in the polarization state of a microwave beam (indicating the presence of a GW) moving in a waveguide about one meter across as shown in Figure 4. Please see Cruise

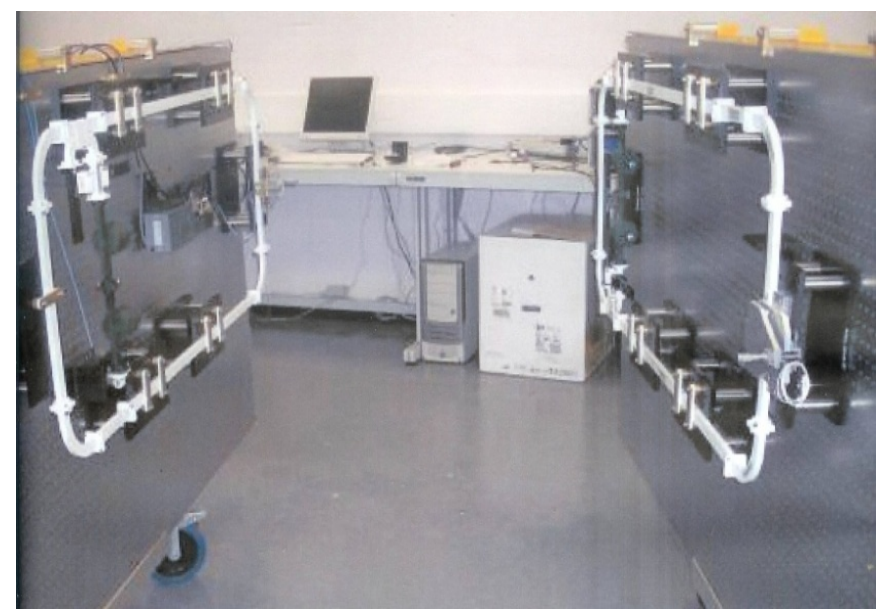

Figure 4. Birmingham University HFRGW Detector. 
(2000) [42]; Ingley and Cruise (2001) [43] and Ingley (2005) [44]. It is expected to be sensitive to HFRGWs having spacetime strains of $\mathrm{A} \sim 2 \times 10^{-13} / \sqrt{\mathrm{Hz}}$, where $\mathrm{Hz}$ is the GW frequency, and as usual, $\mathrm{A}$ is a measure of the strain or fractional deformation in the spacetime continuum (again dimensionless $\mathrm{m} / \mathrm{m}$ ).

The INFN Genoa HFRGW resonant antenna consists of two coupled, superconducting, spherical, harmonic oscillators a few centimeters in diameter. Please see Figure 5. The oscillators are designed to have (when uncoupled) almost equal resonant frequencies. In theory, the system is expected to have a sensitivity to HFRGWs with size (fractional deformations) of about $\sim 2 \times 10^{-17} / \sqrt{\mathrm{Hz}}$ with an expectation to reach a sensitivity of $\sim 2 \times 10^{-20} / \sqrt{\mathrm{Hz}}$. (Bernard, Gemme, Parodi, and Picasso' (2001) [45]; and Ballantini, R, Bernard, Ph, Chiaveri E., Chincarini A., Gemme G., Losito R., Parodi R. and Picasso E. (2003) [46]). As of this date, however, there is no further development of the INFN Genoa HFRGW detector.

The Kawamura $100 \mathrm{MHz}$ HFRGW detector has been built by the Astronomical Observatory of Japan and essentially a table-top higher frequency LIGO. It consists of two synchronous interferometers exhibiting an arm's length of 75 $\mathrm{cm}$. Please see Figure 6. Its sensitivity is now about $10^{-16} \sqrt{\mathrm{Hz}}$ (Nishizawa et al., 2008) [47]. According to Cruise (2008) of Birmingham University [48] its

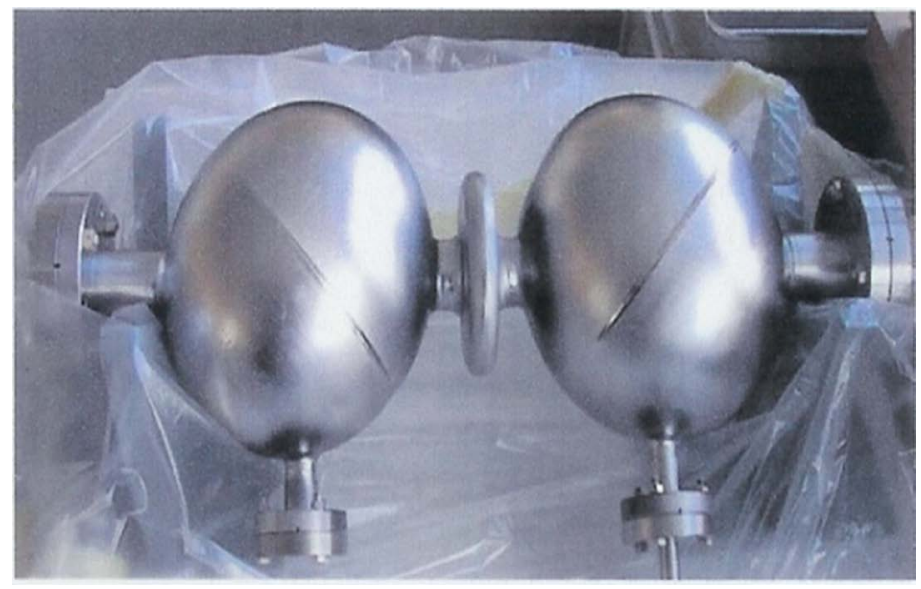

Figure 5. INFN Genoa HFRGW detector.

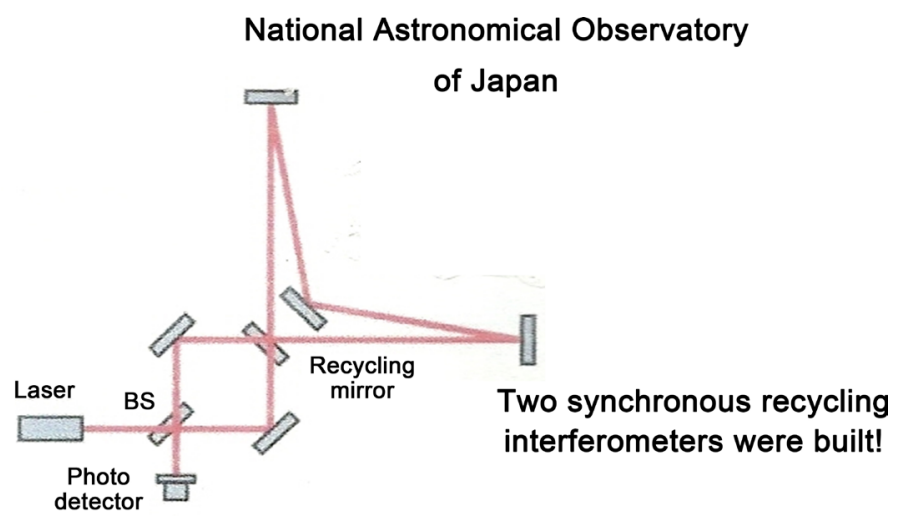

Figure 6. The National Astronomical Observatory of Japan 100MHz Detector. 
frequency is limited to $100 \mathrm{MHz}$ and at higher frequencies, its sensitivity diminishes.

\section{Cruise (2008) Diagram}

Note that all these detectors above can be compared with [49] as to Yag lasers and GW detections, as that the principles for detection are similar (not identical)

\section{The Li-Baker HFRGW Detector}

The Li-Baker HFGW detector was invented by R. M L Baker, Jr. of Transportation Sciences Corporation, California and patented (Baker, 2001) [25] (http://www.gravwave.com/docs/Chinese\%20Detector\%20Patent\%2020081027.p df).

Based upon the theory of Li, Tang and Zhao (1992) [50] termed the Li-effect, the very sensitive detector was proposed by Baker during the period 1995-2000, the patent for it was filed in P. R. China in 2001 [25], subsequently granted in 2007, and preliminary details were published later by Baker, Stephenson and Li (2008) [51].

The Li-Effect, the theoretical basis for the Li-Baker detector, was first published in 1992 [50] and subsequently, some ten peer-reviewed papers have been published concerning it (Li and Tang (1997) [52], Li et al. (2000) [53], Li and Yang (2009) [17], Baker, Li and Li (2006) [54], Li, Baker and Fang (2007) [55], as well as [56], then Baker, Stephenson and Li (2008) [15], and Li et al. (2008) [16]). The capstone paper ( $\mathrm{Li}$, et al., 2008 [16]) presents all of the technical details and is included as APPENDIX B of their paper. The Li Effect is very different from the classical (inverse) Gertsenshtein Effect (Li, 2009 [57]). With the Li-Effect, a gravitational wave transfers energy to a separately generated electromagnetic (EM) wave in the presence of a static magnetic field. That EM wave has the same frequency as the GW (ripple in the spacetime continuum) and moves in the same direction. This is the "synchro-resonance condition," in which the EM and GW waves are synchronized (move in the same direction and have the same frequency).

Although explained in detail in the foregoing references, in order to be more convenient for the reader it is deemed appropriate to summarize the features of the Li-Baker HFRGW detector as follows: The result of the intersection of the parallel and superimposed EM and GW beams, according to the Li-Effect, is new EM photons moving off in a direction perpendicular to the beams and the magnetic field direction. Thus, these new photons occupy a separate region of space (see Figure 7) that can be made essentially noise-free and the synchroresonance EM beam itself (in this case a Gaussian beam) is not sensed there, so it does not interfere with detection of the photons.

The synchro-resonance solution of Einstein's field equations (Li, Baker, Fang, Stephenson and Chen, 2008 pp. 411 to 413) is [16] radically different from the Gertsenshtein (1962) effect (Li, 2009) [57]. The newer Li-Effect solution also uses coupling between EM and gravitational waves (Li, Tang and Zhao, 1992 


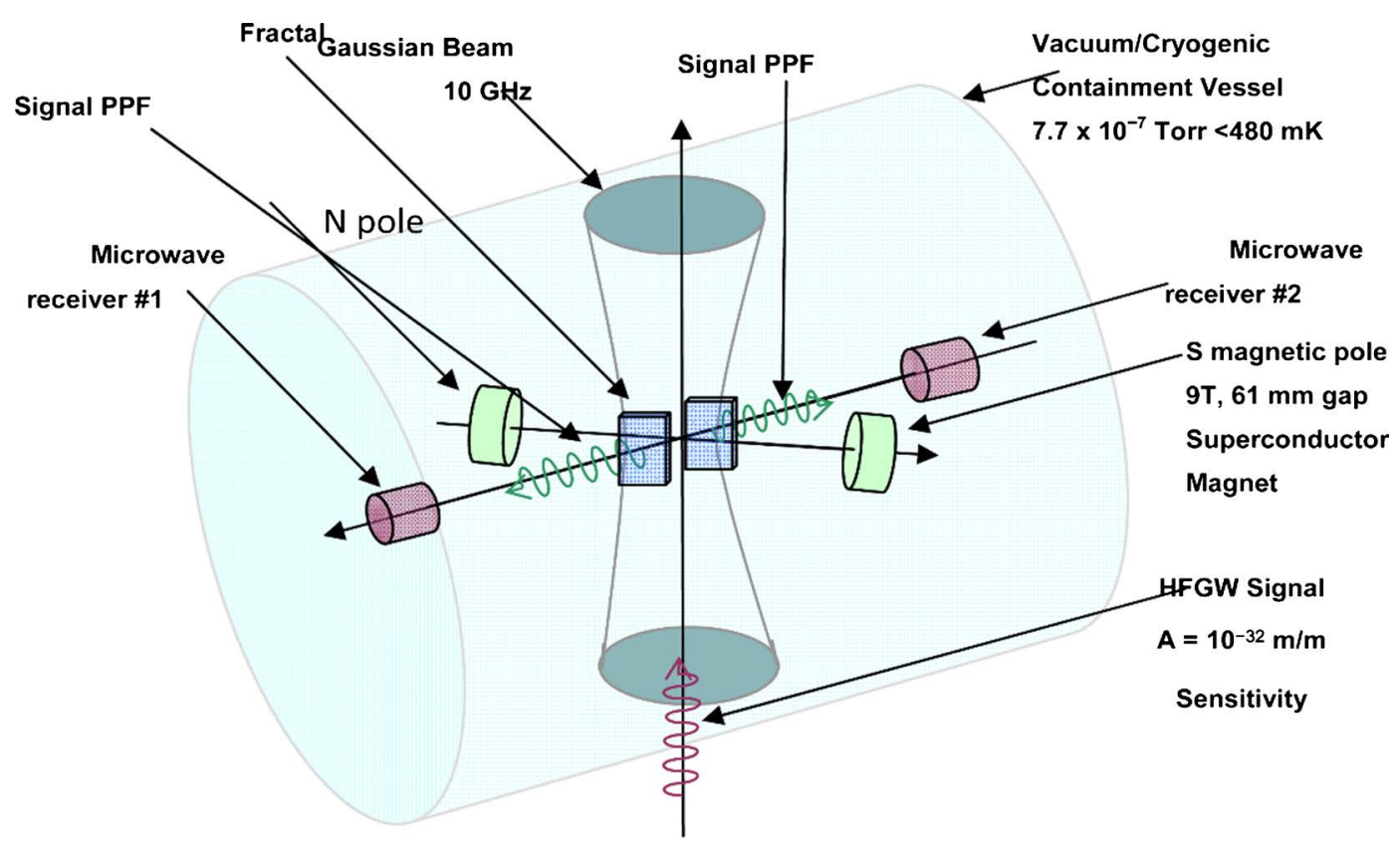

Figure 7. Detection photons sent to locations that are less affected by noise.

[50]) that arises according to the theory of relativity. And from Figure 8 below, a strong static magnetic field in the $y$-direction, $B$, is superimposed upon a GW propagating in the z-direction, as in the inverse Gertsenshtein effect. However, with the Li-Effect, there is an additional focused microwave beam ("Gaussian beam") at the expected frequency, phase and bandwidth of the HFGWs in the same direction ( $\mathrm{z}$ ) as the GW (as shown in Figure 8).

Unlike the Gertsenshtein effect [57], and a similar situation reported the most useful for low-frequency gravitational waves as reported by Gertsenshtein in 1963 [58], a first-order perturbative photon flux (PPF), comprising the detection photons, will be generated in the $\mathrm{x}$-direction. Since there is a 90-degree shift in direction, there is little crosstalk between the PPF and the superimposed EM wave (Gaussian beam), so the PPF signal can be isolated and distinguished from the effects of the Gaussian beam, enabling detection of the GW.

Here's how it works:

1) The perturbative photon flux (PPF), which signals the detection of a passing gravitational wave (GW), is generated when the two waves (EM and GW) have the same frequency, direction and phase. This situation is termed "synchroresonance". These PPF detection photons are generated as the EM wave propagates along its z-axis path, which is also the path of the GWs, as shown in Figure 8.

2) The magnetic field is in the y-direction. According to the Li-Effect, the PPF detection photon flux (also called the "Poynting Vector") moves out along the $\mathrm{x}$-axis in both directions.

3) The signal (the PPF) and the noise, or background photon flux (BPF) from the Gaussian beam have very different physical behaviors. The BPF (background noise photons) are from the synchro-resonant EM Gaussian beam and move in 


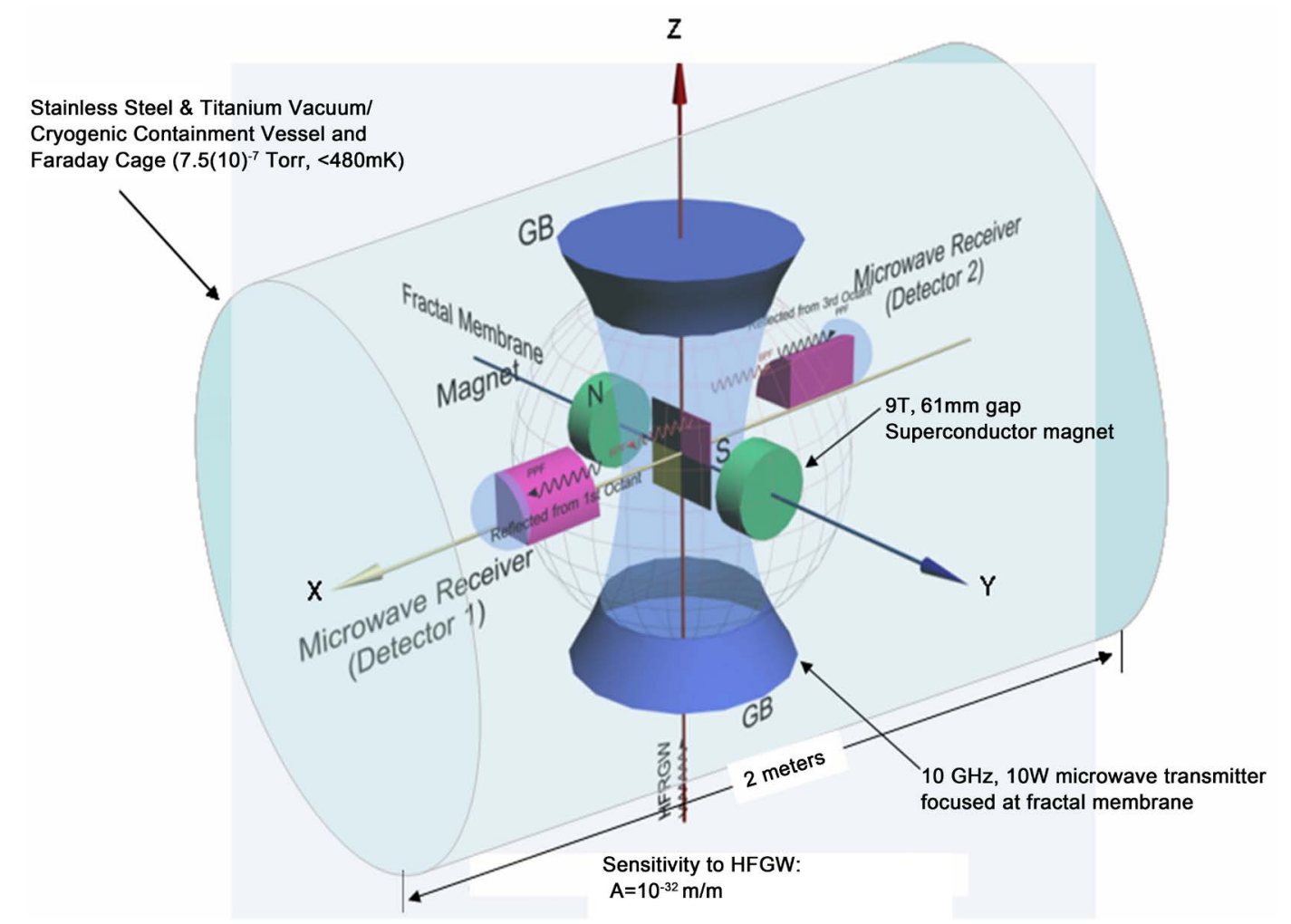

Figure 8. Schematic of ultra-sensitive Li-baker HFRGW detectors.

the z-direction, whereas the PPF (signal photons) move out in the $\mathrm{x}$-direction along the $\mathrm{x}$-axis.

4) The PPF signal can be intercepted by electromagnetic-interference-shielded microwave receivers located on the $\mathrm{x}$-axis (isolated from the synchro-resonance Gaussian EM field, which is along the z-axis). In addition, isolation is further improved by cooling the microwave receiver apparatus to reduce thermal noise background.

The resultant efficiency of detection of HFRGWs utilizing the Li effect is $10^{25}$ times greater than from the inverse Gertsenshtein effect (p. 21, Eardley, 2008 [59]), which has been exploited in some previously proposed HFRGW detectors, e.g., in the JASON Report and in Li (2009) [57]. The proposed novel Li-Baker detection system is shown schematically in Figure 8. The detector is sensitive to HFRGWs directed along the $+\mathrm{z}$-axis, and the precise geometrical arrangement of the major components around this axis is the key to its operation.

The detector, shown in Figure 8, has five major components:

1) A Gaussian (focused, with minimal side lobes) microwave beam (GB) is aimed along the $+\mathrm{z}$-axis at the same frequency as the intended HFRGW signal to be detected (Yariv, 1975 [60]), typically in the $\mathrm{GHz}$ band, and also aligned in the same direction as the HFRGW to be detected. The microwave transmitter's horn antenna is not shown, but would be located on the $-\mathrm{z}$ axis.

2) A static magnetic field $B$, generated by two powerful magnets (typically using powerful superconductor magnets such as those found in a conventional 
MRI medical body scanner), is directed along the y-axis.

3) Two paraboloid-shaped reflectors, which are formed from "fractal membranes" (Wen et al., 2002 [61]; Hou et al., 2005 [62]), are located in the y-z plane at the origin of the coordinate system to aim and focus the detection photons at diffraction limited spot antennas connected to two microwave receivers. These reflectors are segmented (similar to a Fresnel lens) and located back-to-back in the $y-z$ plane. They are thin enough (less than a centimeter thick in the $\mathrm{x}$-direction) to not block the $\mathrm{z}$-directed Gaussian beam. These microwave reflectors reflect the $\mathrm{x}$-directed detection photons (PPF) and reject the $\mathrm{z}$-directed Gaussian-beam photons, which move parallel to the surface of the reflectors in the $y$-z plane.

4) High-sensitivity shielded microwave receivers are located at each end of the $\mathrm{x}$-axis.

5) Interior noise from thermal photon generation is eliminated by cooling the Li-Baker detection apparatus to below $\sim 48 \mathrm{mK}$ (0.048 Kelvin). There are effectively no thermal photons at $10 \mathrm{GHz}$ and higher frquencies. Noise from the interior background photon flux (BPF) from the EM Gaussian beam is reduced to a negligible level by moving the receivers out to the side about a meter away from the EM beam and by a series of superconductor or microwave absorbent baffles to "shade" the receivers. Stray EM resulting from scattering of particulate matter near the apparatus and possible dielectric dissipation can be effectively suppressed by evacuating the apparatus to about $7.5 \times 10^{-7}$ Torr (a rather high vacuum).External noise is eliminated by the use of a steel and titanium cryogenic containment vessel surrounding the low-temperature Li-Baker detection apparatus. Stray noise spillover and diffraction that still manages to get reflected onto the detectors will create the shot noise, but such noise could be filtered out by pulse-modulating the magnetic field (Woods, Baker, Li and Beckwith, 2011) [63]. The idea, suggested by Baker, is that when the pulse-modulated magnetic field is "off" only the stray spillover noise and not the HFGW signal is present. Therefore, it can be effectively subtracted from the noisy signal, leaving only the HFGW signal and the sensitivity of the Li-Baker detector is thereby greatly improved.

\section{Conclusions}

Clearly, much observational data analysis needs to be accomplished both utilizing existing data and data to be obtained with the proposed Li-Baker detector. If it is found that high-accuracy Muon decay time measurement shows a reduction in decay time, then that data could be correlated with the current speed of time as in (Baker, 2019) [27] [30]. In fine it is concluded that high-frequency relic gravitational wave (HFRGW) detectors be developed, especially the Li-Baker HFRGW detector, in the gigahertz and higher frequency range. Thereby producing cosmological, primordial observational data especially generated during the first second after the beginning of our Universe. Up to now, cosmological 
measurements have led to the theoretical introduction of dark matter and dark energy. An alternative working hypothesis or theory of the rollout of our Universe in spacetime from ultra-small Planck Length space dimensions and ultra-short time Planck Time to current values is suggested. This new innovative theory does not require the introduction of dark matter or dark energy and may account for dwarf galaxies, surprisingly "modern" cosmic clouds (Banados, 2019) [64], "Oh My God" (OMG) high-energy particles, and other unexplained peculiar features of our early Universe. It is suggested that the speed of time also be estimated based upon the rate of separation of cosmological objects (Hubble number) and galactic rotational rate when attributed to the speed of time not dark energy or dark matter. A fundamental equation is presented that links a change in the speed of time and the speed of the lengthening of dimensions, as they slow from their initial values, in order to preserve the constancy of the speed of light. The working hypothesis or theory not only encourages the development of HFRGW detectors, especially the Li-Baker, but also challenges theoreticians to enhance the mathematical rigor of the rollout theory as the recommended HFGRGW detector collects relic, primordial data. It is recommended that plans and detailed specifications for the Li-Baker HFRGW detector be prepared in order to expedite its fabrication.

We assert that the astrophysics benefits of HFGW would among other things be linked to ascertaining the probability of new polarization states in GW detections, as given by Fangyu Li et al. (2018) [65] and in addition, HFGW may enable investigating the fundamental constants of nature as stated by Barrow et al. [66] which is a window into the possibility of new physical theories of gravity as given by Professor Corda, in a 2009 paper [67] i.e. not only should the fundamental constants of nature be reviewed as well as their genesis in early universe cosmology are of striking import. In particular, the author's point to Dr. Lis 2018 [65],

$$
h_{u v}(t)=\left(\begin{array}{cccc}
0 & 0 & 0 & 0 \\
0 & A_{\oplus}+A_{b} & A_{\otimes} & A_{x} \\
0 & A_{\circledast} & -A_{\oplus}+A_{b} & A_{y} \\
0 & A_{x} & A_{y} & \sqrt{2} A_{l}
\end{array}\right)_{u v} \cdot \exp \left(i k_{g} z-i \omega_{g} t\right)
$$

As well as [65],

$$
h_{i j}^{k \neq 0}(t)=\left(\begin{array}{ccc}
h^{+}-\frac{h^{l, O}}{2} & h^{\times} & h_{1}^{l} \\
h^{\times} & -h^{+}-\frac{h^{l, O}}{2} & h_{2}^{l} \\
h_{1}^{l} & h_{2}^{l} & h^{l, O}
\end{array}\right)_{i j} \cdot \cos \left(m_{k} \cdot c^{2} \cdot t\right)
$$

The off diagonal contributions, at one goal, would be probed as of Barrows suppositions as to contributions due to setting the initial space-time constants, and would also enable a full investigation as to the scalar-tensor gravity theories 
brought up by Professor Corda in his 2009 [67] outstanding presentation. We also assert that HFGW are the most direct way into review of these fundamental polarization state contributions as Equations (3) and (4) are straight from Dr. Li et al. 2018 [65] which the author views as of fundamental import as to the foundations of gravitational waves at the start of the early universe. Mind you this will be a rich investigation into the fundamental structures of space time which we assert will be of invaluable import to the development of gravitational wave astronomy.

All this started with many refinements beyond what was presented in [68] which is an extension of lasers and interferometry, i.e. we have taken the search implied by these same tools and extended their reach. We also argue this would be able to compliment [69] which albeit vastly improved over the first generation of LIGO but was still functionally not too effective for GW above 1000 Hertz.

\section{Acknowledgements}

The authors, in particular owe a great deal to Gary Stephenson, and to Clive Woods, of LSU for their understanding of electronics which may enable full utilization of and development of sensing applications of HFRGWs to not only astrophysics and cosmology, but global communications, and other endeavors. Of course, the debt this project owes to Dr. Fangyu Li of Chongqing University cannot be over stated, as to his through development of theoretical sensing requirements for this hardware system. Further developments of this document await input from other members of the GravWave team (http://www.gravwave.com/). Also, Amara Angelica provided decisive image enhancement assistance which Dr. Andrew Beckwith used to achieve maximum clarity and resolution to four of this paper's figures. Big thank you to Amara Angelica because Andrew Beckwith had no idea to work with the needed file technology to full effect.

\section{Conflicts of Interest}

The authors declare no conflicts of interest regarding the publication of this paper.

\section{References}

[1] Dodelson, S., et al. (2009) The Origin of the Universe as Revealed through the Polarization of the Cosmic Microwave Background. https://arxiv.org/abs/0902.3796

[2] Griskchuck, L. (2006) Relic Gravitational Waves and Cosmology. https://arxiv.org/pdf/gr-qc/0504018.pdf

[3] Grishchuk, L.P. (2008) Discovering Relic Gravitational Waves in Cosmic Microwave Background Radiation. In: Ciufolini, I. and Matzner, R., Eds., Proceedings of the School, Springer, Berlin, 75-85.

[4] Grishchuk, L.P. (2007) High-Frequency Relic Gravitational Waves, Their Detection and New Approaches. Proceedings of the HFGW2 Workshop, Austin, 19-21 September 2007. http://earthtech.org/hfgw2

[5] Shawhan, P.S. (2004) Gravitational Waves and the Effort to Detect Them. American 
Scientist, 92, 356. https://doi.org/10.1511/2004.48.938

[6] Shoemaker, D. (2008) Context and Summary, Advanced LIGO.

https://dcc.ligo.org/public/0151/T1800133/004/T1800133-instrument-science-white -v4.pdf

[7] Beckwith, A.W. (2009) Relic High Frequency Gravitational Waves, Neutrino Physics, and Icecube. Proceedings of the Space, Propulsion and Energy Sciences International Forum, Huntsville, 24-27 February 2009, 564-570. https://doi.org/10.1063/1.3115566

[8] Beckwith, A.W. (2009) Relic High Frequency Gravitational Waves from the Big Bang and How to Detect Them. Proceedings of the Space, Propulsion and Energy Sciences International Forum, Huntsville, 24-27 February 2009, 571. https://doi.org/10.1063/1.3115567

[9] Beckwith, A.W. (2009) Entropy Growth in the Early Universe. http://www.gravwave.com/docs/Entropy\%20_provisional\%20draft $\% 20 \_$Andrew $\% 2$ 0Beckwith.pdf

[10] Beckwith, A.W. (2008) Implications for the Cosmological Landscape: Can Thermal Inputs from a Prior Universe Account for Relic Graviton Production? AIP Conference Proceedings, 969, 1091. https://doi.org/10.1063/1.2844947

[11] Ng, J.Y. (2012) MoNDian Dark Matter, Entropic Gravity, and Infinite Statistics. https://arxiv.org/abs/1212.6433

[12] Ng, Y. (2007) Jack Holographic Foam, Dark Energy and Infinite Statistics. Physics Letters B, 657, 10-14. https://doi.org/10.1016/j.physletb.2007.09.052

[13] Ng, Y.J. (2008) Spacetime Foam: From Entropy and Holography to Infinite Statistics and Nonlocality. Entropy, 10, 441-461. https://doi.org/10.3390/e10040441

[14] Beckwith, A.W. (2007) Entropy Growth in the Early Universe and Confirmation of Initial Big Bang Conditions (Why the Quark-Gluon Model Is Not the Best Analogy). https://arxiv.org/ftp/arxiv/papers/0712/0712.0029.pdf

[15] Baker, R.M.L., Stephenson, G.V. and Li, F. (2008) Proposed Ultra-High Sensitivity HFGW Detector. Proceedings of Space Technology and Applications International Forum, NM, USA, 2-17 April 2008, 1045-1054.

[16] Li, F.Y., Baker, R.M.L., Fang, Z., Stephenson, G.V. and Chen, Z. (2008) Perturbative Photon Fluxes Generated by High-Frequency Gravitational Waves and Their Physical Effects. The European Physical Journal C, 22, 1-39.

http://www.gravwave.com/docs/Li-Baker\%206-22-08.pdf

[17] Li, F.Y. and Yang, N. (2009) Phase and Polarization State of High-Frequency Relic Gravitational Waves. Chinese Physics Letters, 33, Article ID: 100402. https://doi.org/10.1088/0256-307X/26/5/050402

[18] Lemley, B. and Fink, L. (2002) Guth's Grand Guess. Discover Magazine, 23, 1/8-8/8.

[19] Brandenberger, R.H. (2011) Introduction to Early Universe Cosmology. 4th International Conference on Fundamental Interactions, 124, 1-70. https://doi.org/10.22323/1.124.0001

[20] Shields, P. and Rangarjan, N. (2013) A Playbook for Research Methods: Integrating Conceptual Frameworks and Project Management. New Forums Press, Stillwater, Chapter 5.

[21] Newton, I. (1822) Philosophiae Naturalis Principia Mathematica. Kessinger Publishing, London.

[22] Newton, I. (2008) The Principia: Mathematical Principles of Natural Philosophy. Snowball Publishing Edition, London. 
[23] Einstein, A. (1917) Cosmological Considerations in the General Theory of Relativity. Königlich Preussische Akademie der Wissenschaften, Berlin. (In German)

[24] Baker, R.M.L. and Noble, F. (2000) United States Patent Number 6,160,336 Peak Power Energy Storage Device and Gravitational Wave Generator. December 12, Section on Space Time Universe Geometry.

[25] Baker, R.M.L. (2001) 2001 Peoples Republic of China Patent Number 01814223.0 Gravitational Wave Generator and Detector. Filed July 13, 2001, Granted September 19, 2007.

[26] Baker, R.M.L. (2017) Gravitational Waves: The World of Tomorrow, a Primer, with Exercises. Infinity Publishing, PA, USA, $234 \mathrm{p}$.

[27] Baker, R.M.L. (2018) Analyses of the Speed of Time Based on Muon Lifetime-Decay as a Transient Time. Annual Meeting of the American Association for the Advancement of Science, Austin, 18 February 2018.

[28] Senovilla, J. (2007) Is Time Slowing Down? New Scientist, Issue 2635, 5-22, December 22.

[29] Mars, M., Senovilla, J. and Vera, R. (2008) Is the Accelerated Expansion Evidence of a Forthcoming Change of Signature on the Brane? Physical Review D, 77, Article ID: 027501. https://doi.org/10.1103/PhysRevD.77.027501

[30] Baker, R.M.L. (2019) A Working Hypothesis on the Muon-Decay Time Shortening and Time. Journal of Space Science \& Technology, 25, 60-77. https://doi.org/10.15407/knit2019.03.060

[31] Baker, R.M.L. and Baker, B.S. (2019) Brainwaves, Gravitational Waves, New Theory of Our Universe and Exobiology. https://youtu.be/3UcJfvfoQIQ

[32] Alam, S., et al. (2017) The Clustering of Galaxies in the Completed SDSS-III Baryon Oscillation Spectroscopic Survey: Cosmological Analysis of the DR12 Galaxy Sample. Monthly Notices of the Royal Astronomical Society, 470, 2617-2652.

[33] Reiss, A.G., et al. (2019) Large Magellanic Cloud Cepheid Standards Provide a 1\% Foundation for the Determination of the Hubble Constant and Stronger Evidence for Physics beyond ACDM. Astrophysical Journal, 876, 85.

[34] Herrick, S. (1971) Astrodynamics. Van Nostrand Reinhold, New York.

[35] Baker, R.M.L. and Makemson, M.W. (1967) An Introduction to Astrodynamics. Second Edition, Academic Press, New York.

[36] Baker, R.M.L. (1967) Astrodynamics, Applications and Advanced Topics. Academic Press, New York, 484.

[37] Braginsky, V.B., Grishchuk, L.P., Doroshkevich, A.G., Zeldovich, Ya.B., Noviko, I.D. and Sazhin, M.V. (1974) Electromagnetic Detectors of Gravitational Waves. Soviet Physics-JETP, 38, 865.

[38] Pegoraro, F., Radicati, L.A., Bernard, Ph. and Picasso, E. (1978) Electromagnetic Detector for Gravitational Waves. Physics Letters A, 68, 165-168. https://doi.org/10.1016/0375-9601(78)90792-2

[39] Rudenko, V.N. and Sazhin, M.V. (1980) Laser Interferometer as a Detector of Gravitational Waves. Kvantovaya Electronica, 7, 2344-2355.

[40] Tobar, M.E. (1995) Characterizing Multi-Mode Resonant-Mass Gravitational Wave Detectors. Journal of Applied Physics, 28, 1729-1736. https://doi.org/10.1088/0022-3727/28/8/025

[41] Tobar, M.E. (1999) Microwave Parametric Transducers for the Next Generation of Resonant-Mass Gravitational Wave Detectors. Department of Physics Publication, 
the University of Western Australia, Newlands.

[42] Cruise, A.M. (2001) An Electromagnetic Detector for Very-High-Frequency Gravitational Waves. Classical and Quantum Gravity, 17, 2525. https://doi.org/10.1088/0264-9381/17/13/305

[43] Ingley, R.M.J. and Cruise, A.M. (2001) An Electromagnetic Detector for High Frequency Gravitational Waves. 4th Edoardo Amaldi Conference on Gravitational Waves, Perth, July 2001, 21-25.

[44] Ingley, R.M.J. (2005) Implementation and Cross Correlation of Two High Frequency Gravitational Wave Detectors. PhD Thesis, the University of Birmingham, Birmingham.

[45] Bernard, Ph., Gemme, G., Parodi, R. and Picasso, E. (2001) A Detector of Small Harmonic Displacements Based on Two Coupled Microwave Cavities. Review of Scientific Nuclear Instruments, 72, 2428-2437. https://doi.org/10.1063/1.1366636

[46] Ballantini, R., Bernard, Ph., Chiaveri, E., Chincarini, A., Gemme, G., Losito, R., Parodi, R. and Picasso, E. (2003) A Detector of High Frequency Gravitational Waves Based on Coupled Microwave Cavities. Classical and Quantum Gravity, 20, 3505. https://doi.org/10.1088/0264-9381/20/15/316

[47] Nishizawa, A., Kawamura, S., Akutsu, T., Arai, K., Yamamoto, K., Tatsumi, D., Nishida, E., Sakagami, M., Chiba, T., Takahashi, R. and Sugiyama, N. (2008) Laser-Interferometric Detectors for Gravitational Wave Backgrounds at $100 \mathrm{MHz}$ : Detector Design and Sensitivity. Physical Review D, 77, Article ID: 022002. https://doi.org/10.1103/PhysRevD.77.022002

[48] Cruise, A.M. (2008) Very High Frequency Gravitational Waves. Gravitational Wave Advanced Detector Workshop (GWADW) Elba Conference, 17 May 2008, Slide Presentation 132.

https://indico.pi.infn.it/contributionDisplay.py?contribId=132\&sessionId=13\&conf $\mathrm{Id}=225$

[49] Ottaway, D.J., et al. (1998) A Compact Injection-Locked Nd:YAG Laser for Gravitational Wave Detection. IEEE Journal of Quantum Electronic, 34, 2006-2009. https://doi.org/10.1109/3.720240

[50] Li, F.Y., Tang, M. and Zhao, P. (1992) Interaction between Narrow Wave BeamType High Frequency Gravitational Radiation and Electromagnetic Fields. Acta Physica Sinica, 41, 1919-1928.

[51] Baker, R.M.L., Stephenson, G.V. and Li, F. (2007) Discussion-Focus Paper 1.2 "Proposed Ultra-High Sensitivity High-Frequency Gravitational Wave Detector" for 2nd Invitational HFGW Workshop September 17-20, 2007, Institute for Advanced Studies at Austin, Texas. 27 June 27, 2007, Preliminary Draft. http://www.gravwave.com/docs/Paper1.2\%206-25\%20Ultra-HighSensitivity.pdf

[52] Li, F.Y. and Tang, M.X. (1997) Positive Definite Problem of Energy Density and Radiative Energy Flux for Pulse Cylindrical Gravitational Wave. Acta Physica Sinica, 6, 321-333. https://doi.org/10.1088/1004-423X/6/5/001

[53] Li, F.Y., Tang, M.-X., Luo, J. and Li, Y.-C. (2000) Electro Dynamical Response of a High Energy Photon Flux to a Gravitational Wave. Physical Review D, 62, Article ID: 044018. https://doi.org/10.1103/PhysRevD.62.044018

[54] Li, F.Y. and Li, R.X. (2006) Ultra-High-Intensity Lasers for Gravitational Wave Generation and Detection. Proceedings of Space Technology and Applications International Forum, New York, 12-16 February 2006, 1249-1258. http://www.drrobertbaker.com/docs/AIP;\%20HFGW\%20Laser\%20Generator.pdf

[55] Li, F.Y. and Baker, R.M.L. (2007) Detection of High-Frequency Gravitational Waves 
by Superconductors. International Journal of Modern Physics, 21, 3274-3278. https://doi.org/10.1142/S0217979207044366

[56] Li, F.Y., Baker, R.M.L. and Fang, Z. (2007) Coupling of an Open Cavity to a Microwave Beam: A Possible New Scheme for Detecting High-Frequency Gravitational Waves. AIP Conference Proceedings, 880, 1139-1147. https://doi.org/10.1063/1.2437560

[57] Li, F.Y. (2009) Response to Adverse JASON Report on Li-Effect. http://www.gravwave.com/docs/Li\%20response\%20to\%20JASON.pdf

[58] Gertsenshtein, M.E. and Pustovoit, V.I. (1963) On the Detection of Low Frequency Gravitational Waves. Journal of Experimental and Theoretical Physics, 16, 433.

[59] Eardley, et al. (2008) High Frequency Gravitational Waves. JSR-08-506, October, the JASON Defense Science Advisory Panel and Prepared for the Office of the Director of National Intelligence.

[60] Yariv, A. (1975) Quantum Electronics. 2nd Edition, Wiley, New York.

[61] Wen, W.J., Zhou, L., Li, J.S., Ge, W.K., Chan, C.T. and Sheng, P. (2002) Sub-Wavelength Photonic Band Gaps from Planar Fractals. Physical Review Letters, 89, Article ID: 223901. https://doi.org/10.1103/PhysRevLett.89.223901

[62] Hou, B., Xu, G., Wong, H.K. and Wen, W.J. (2005) Tuning of Photonic Bandgaps by a Field-Induced Structural Change of Fractal Metamaterials. Optics Express, 13, 9149-9154. https://doi.org/10.1364/OPEX.13.009149

[63] Woods, C.R., Baker, R.M.L., Li, F., Stephenson, G.R., Davis, E.W. and Beckwith, A.W. (2011) A New Theoretical Technique for the Measurement of High-Frequency Relic Gravitational Waves. Journal of Modern Physics, 2, 498-518.

[64] Banados, Edwardo, et al. (2019) Cosmic Clouds Lived and Died More Quickly than Thought. Astrophysics Journal, 885, 59.

[65] Li, F.-Y., Wen, H., Fang, Z.-Y., Li, D. and Zhang, T.-J. (2018) Electromagnetic Counterparts of High-Frequency Gravitational Waves Having Additional Polarization States: Distinguishing and Probing Tensor-Mode, Vector-Mode and Scalar-Mode Gravitons. https://arxiv.org/pdf/1712.00766.pdf

[66] Barrow, J.D. (2002) The Constants of Nature; from Alpha to Omega-The Numbers That Encode the Deepest Secrets of the Universe. Pantheon Books, New York.

[67] Corda, C. (2009) Interferometric Detection of Gravitational Waves: The Definitive Test for General Relativity. International Journal of Modern Physics D, 18, 2275-2282. https://arxiv.org/abs/0905.2502 https://doi.org/10.1142/S0218271809015904

[68] Chincarini, A. and Gemme, G. (2003) Micro-Wave Based High-Frequency Gravitational Wave Detector. Gravitational-Wave Conference, 6-9 May 2003, Paper HFGW03-103.

[69] LIGO Scientific Collaboration (2011) A Gravitational Wave Observatory Operating beyond the Quantum Shot-Noise Limit. Nature, 7, 962-965.

https://doi.org/10.1038/nphys2083 